\title{
Numerical study on the effect of the die compression angle on extrusion forming of plastic pipe
}

\author{
Zhong Ren ${ }^{1}$ a, Xingyuan Huang ${ }^{2}$, Zhihua Xiong ${ }^{1}$ \\ ${ }^{1}$ Key Laboratory of Optic-electronics and Communication, Jiangxi Science and Technology Normal University,330038 Nanchang, China \\ ${ }^{2}$ School of Mechanical and Electrical Engineering, Nanchang University, 330031 Nanchang, China
}

\begin{abstract}
The effect of compression angle of die on the extrusion forming of plastic pipe was numerically studied by using the finite element method. Based on the geometric models of plastic pipes with four different compression angles, the extrudate swell results of plastic pipe under the different compression angles are numerically simulated. Numerical results show the extrudate swell phenomenon for the little compression angles are larger than that of the large compression angles. To eliminate the extrudate swell effect and keep the wear-resistant strength of the plastic pipe, the gas-assisted extrusion method was used in this paper. The numerical results fully show that the shear stress of melt was greatly eliminated and the high pressure was still maintained by using the gas-assisted method.
\end{abstract}

\section{INTRODUCTION}

Plastic pipe is a kind of important product widely used in many fields, such as water supply and drainage, gas transmission, medical catheter, automobile oil-way, and daily necessaries. The manufacture of the plastic pipe is usually performed by the extrusion forming method of the polymer melt [1]. At the same time, the extrusion forming of the plastic pipe is usually achieved by the aid of the metal die with a certain of cross-shape. The quality of extruded plastic pipe is directly depended on the channel structure of the metal die [2], such as the channel length, channel width, compression angle, etc. In the practice, the manufacture of the plastic pipe should follow some standards including the size precision of pipe's diameter and wall thickness, the compressive strength, wear-resistant strength etc. To achieve these indexes, the metal die should be reasonably designed and manufactured. The numerical simulation is a good method of designing the die for the plastic pipe [3], which can greatly improve the design efficiency and save the cost of the die manufacture. In this paper, to study the effect of die's compression angle on the traditional extrusion forming of the plastic pipe, the numerical method based on the fluid dynamic software Polyflow was used. Numerical results show that for the traditional extrusion forming of plastic pipe, although the extrudate swell decreases with the increasing of the compression angle, the compressive and wear-resistant strength of the plastic pipe will weak because the pressure of polymer melt decreases. Therefore, the compression angle of die can't be too large. In order to overcome the extrudate swell phenomenon of the traditional extrusion forming of the plastic pipe induced by the pressure effect, the gas- assisted extrusion method $[4,5]$ was used and compared with the traditional extrusion forming in this paper. Results show that the gas-assisted method well solve the extrudate swell problem of traditional extrusion for plastic pipe.

\section{NUMERICAL SIMULATION}

\subsection{Geometric models and meshes}

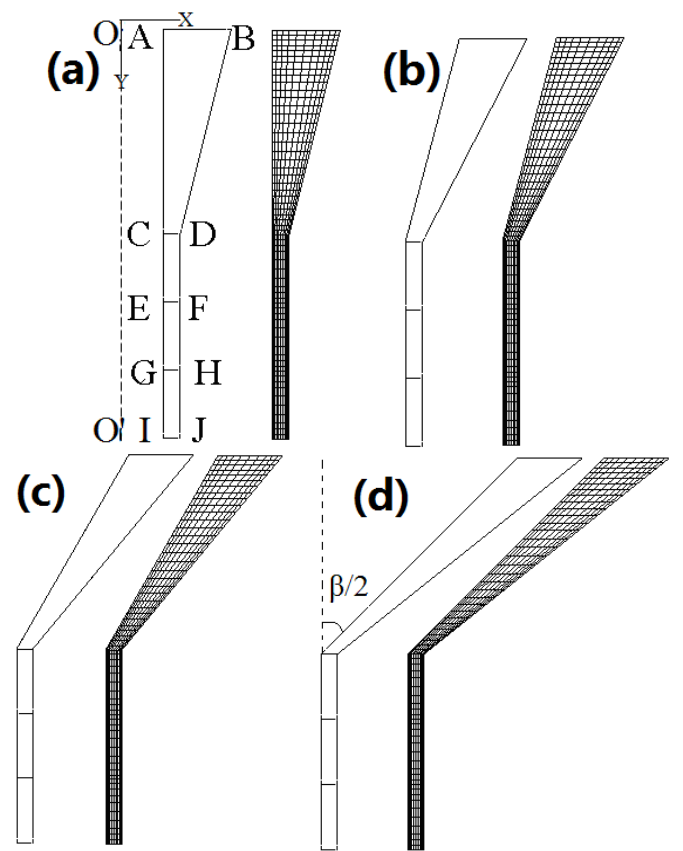

Figure 1. The geometric models and meshes of plastic pipes with different compression angles. (a) $\beta=0$; (b) $\beta=30$; (c) $\beta=60$; (d) $\beta=90$.

\footnotetext{
a Corresponding author: renzhong0921@163.com
} 
Figure 1 shows the geometric models and meshes of plastic pipes with four different compression angles $(\beta)$. In Figure 1(a), the length and width of compression section $(\mathrm{AC}$ and $\mathrm{AB}$ ) are $30 \mathrm{~mm}$ and $10 \mathrm{~mm}$, respectively. The length of shaping zone (CE and EG) are all 10mm. The length of plastic pipe outside die is $10 \mathrm{~mm}$. The inner diameter and wall thickness of the plastic pipe (OI and IJ)are $5 \mathrm{~mm}$ and $2.5 \mathrm{~mm}$, respectively. The quadrilateral mesh structure was used because the geometric model of plastic pipe is regular. To improve the numerical precision, the mesh was refined near the boundaries and interfaces.

\subsection{Governing equations}

To get the numerical results and physical fields distributions, the reasonable hypotheses should be satisfied. Firstly, the melt of plastic pipe was regarded as the iso-thermal, steady, laminar and Non-Newtonian flow. Secondly, the gravity and inertia forces of melt were neglected due to the properties of high viscosity and low flow velocity. Based on the hypotheses, the governing equations can be expressed as follows,

$$
\begin{gathered}
\nabla \cdot v=0 \\
\nabla p-\nabla \cdot \tau=0
\end{gathered}
$$

where $\nabla$ is Hamilton operator, $v$ is the velocity, $p$ is the pressure, $\tau$ is the extra stress tension.

In the numerical simulations, PTT constitutive model [6] was used to describe the viscous-elastic properties of plastic pipe's melt, i.e.,

$$
\exp \left[\frac{\varepsilon \lambda}{\left(1-\eta_{r}\right)} \operatorname{tr}(\tau)\right] \tau+\lambda\left[\left(1-\frac{\xi}{2}\right) \tau+\frac{\xi}{2} \tau\right]=2\left(1-\eta_{r}\right) \eta D
$$

where $\lambda$ is the relaxation time, $\varepsilon$ and $\xi$ are the parameters of the melt correlated with the material tensile and the shear characteristics, respectively. $\tau$ is the upper convected derivative of the extra stress tensor $\tau$. $D$ is the strain-rate of the tensor. $\eta_{r}=\eta_{2} / \eta$ is the viscosity ratio, $\eta$ is the total viscosity of the melt, $\eta_{1}$ is the Non-Newtonian component viscosity of the melt, $\eta_{2}$ is the Newtonian viscosity component of the melt.

\subsection{Boundary conditions}

(1) inlet: In the Figure 1(a), AB is the inlet boundary of melt. Supposed that the melt flow is already fulldeveloped, the following relationship can be satisfied, i.e., $v_{\mathrm{x}}=0, \partial v_{y} / \partial y=0$. where $v_{\mathrm{x}}, v_{\mathrm{y}}$ are the flow velocities of melt at the direction of $x$ and $y$ coordination, respectively.

(2) Wall: $\mathrm{BD}, \mathrm{DH}, \mathrm{AC}$, and $\mathrm{CG}$ are the wall of die's mandrel. The no-slip condition was used, i.e., $v_{s}=v_{n}=0$. where $v_{\mathrm{s}}$ and $v_{\mathrm{n}}$ are the tangential and normal flow velocities, respectively. However, for the gas-assisted extrusion, the gas-assisted sections of die (FH and EG), the full slip condition was used, i.e., $v_{n}=f_{s}=0$. where $f_{\mathrm{s}}$ is the tangential shear stress of melt.

(3) Free boundary: GI and HJ are the free boundaries. The following relationship should be satisfied, i.e., $f_{n}=0, f_{s}=0$ and $v_{n}=0$. where $f_{\mathrm{n}}$ is the normal stress of melt. Moreover, the surface tensions on the free boundaries were ignored.

(4) Exit: Without any tangential and normal forces were imposed on the exit of melt, i.e., $f_{n}=f_{s}=0$.

\subsection{Material parameters}

The constitutive parameters of PTT model for the plastic pipe are shown in Table 1.

Table 1. The parameters of PTT constructive model

\begin{tabular}{llllll}
\hline Parameters & $\eta($ Pa.s $)$ & $\lambda(\mathrm{s})$ & $\varepsilon$ & $\xi$ & $\eta_{\mathrm{r}}$ \\
\hline Values & 2700 & 0.2 & 0.23 & 0.18 & 0.12 \\
\hline
\end{tabular}

\section{NUMERICAL RESULTS AND ANALYSES}

\subsection{Effect of compression angle on the extrudate swell of plastic pipe}

On the inlet boundary, the flow volume rate $1.0 \times 10^{-6} \mathrm{~m}^{3} / \mathrm{s}$ was imposed. The extrudate swell rations of plastic pipes under the different compression angles are obtained by using the finite element computing, which are shown in Figure 2.

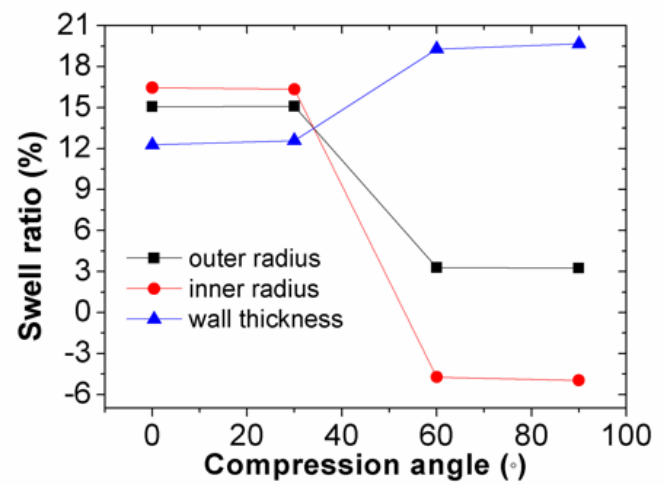

Figure 2. Swell ratios of extruded plastic pipes under the different compression angles

From Figure 2, it can be seen that the swell ratios of outer and inner radius for plastic pipe at the little compression angles $\left(0-30^{\circ}\right)$ are larger than that of the larger compression angles $\left(60-90^{\circ}\right)$. That is, although the extrudate swell phenomenon of diameters for plastic pipe can be decreased with the increasing of the compression angles, from Figure 2, the swell ratios of the wall thickness increases with the compression angles. Therefore, the choosing of the compression angle should be coupled the extrudate swell of diameters and wall thickness. At the same time, the physical field distributions of plastic pipe's melt under each compression angle should be considered. 


\subsection{Physical field distributions of plastic pipe's melt}

The physical field distributions of plastic pipe's melt with four different compression angles are shown in Figure 3.
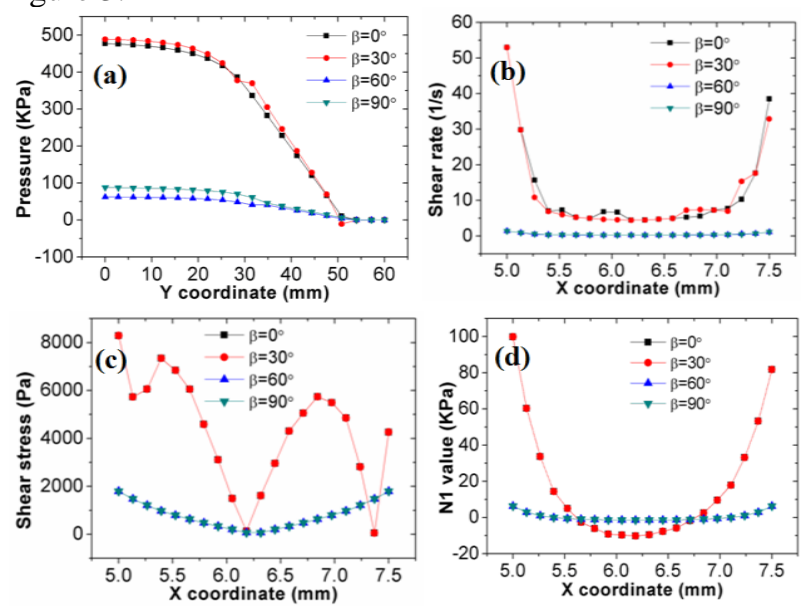

Figure 3. Physical field distributions of plastic pipe's melt with four different compression angles. (a)axial pressure distributions; (b) radial shear rate distributions at the outlet of die; (c)radial shear stress distributions; (d) radial first normal stress difference distributions.

As we know, the extrudate swell effect of melt is directly dependent on the melt's pressure, shear rate, shear stress and the first normal stress difference in the channel. From Figure 3, it can be seen that the pressures, shear rates, shear stresses and the first normal stress differences of melt at the little compression angles (e.g., $\beta=0$, and $30^{\circ}$ ) are too much larger than that of the larger compression angles (e.g., $\beta=60$, and $90^{\circ}$ ). That is, the extrudate swell phenomenon of plastic pipe at the little compression angles are more serious than that of the larger compression angles. However, in the practice, the compressive and wear-resistant strength of the plastic pipe need to reasonably increase the pressure of melt. Therefore, the compression angle of die should be chosen between $30^{\circ}$ and $60^{\circ}$. In general, the compression angle is about $45^{\circ}-60^{\circ}$ for the melt with low viscosity. The compression angle is about $30^{\circ}-50^{\circ}$ for the melt with high viscosity.

\subsection{Gas-assisted extrusion forming of the plastic pipe}

To eliminate the extrudate swell effect of the plastic pipe, the gas-assisted method was used in this work. In the numerical simulations, to achieve the gas-assisted extrusion mode, the boundary condition was set to full slip at the gas-assisted sections (EG and $\mathrm{FH}$ ) in Figure 1(a). The plastic pipe extruded by using the traditional extrusion method and the gas-assisted extrusion method are obtained, which are shown in Figure 4.

From Figure 4(a), it can be obviously seen that the extrudate swell phenomenon of plastic pipe was greatly eliminated by using the gas-assisted method. The radial shear stress distributions of melt at the outlet of die were obtained, which are shown in Figure 4(b). From Figure 4(b), it can be found that the radial shear stress of melt is greatly decreased to zero under the aid of the gasassisted mode. At the same time, from Figure 4(a), it can be also seen that the pressure of melt is still high, which will can ensure the compressive and wear-resistant strength of the plastic pipe.
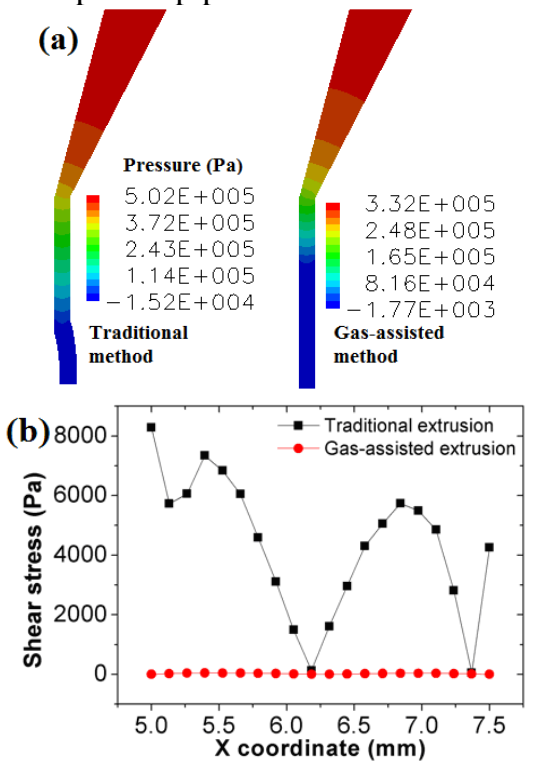

Figure 4. Numerical results by using the traditional extrusion method and the gas-assisted extrusion method

\section{Conclusion}

In this paper, the effect of compression angle of die on the extrusion forming of plastic pipe was numerically studied. Numerical results show that the extrudate swell of plastic pipe are larger for the little compression angles than that of the large compression angles. Not only to eliminate the extrudate swell effect, but also to ensure the compressive and wear-resistant strength of the plastic pipe, the gas-assisted extrusion method was used. The numerical results show that under the help of the gasassisted method, the radial shear stress of melt is nearly decreased to zero, and the pressure of melt is still larger. Therefore, the gas-assisted method has the satisfactory manufacture effect in the extrusion of the plastic pipe.

\section{Acknowledgments}

This work was financially supported by the National Natural Science Foundation of China (51763011), Topnotch talent foundation project of JXSTNU (2014QNBJRC004), and Doctor start-up foundation project of JXSTNU (2017BSQD021).

\section{References}

1. M. Ketata, A. Ayadi, N. Elkissi, et al., Rheol. Acta 56, 341 (2017)

2. Z. Ren, X. Y. Huang, 2016 International Conference on Material Science and Engineering 2016, 0414 (2016) 
3. S V. Ershov, N. M. Trufanova, Materials Science and Engineering Conference Series 2017,012018 (2017)

4. Z. Ren, X. Y. Huang, H. S. Liu, et al., J. Appl. Polym. Sci. 132, 42682 (2015)

5. Z. Ren, X. Y. Huang, Mater. Sci. Forum 2016189 (2016)

6. N.P. Thien, R.I. Tanner, J. Non-Newton Fluid. Mech. 2, 353 (1977) 